\title{
WALKING THE TALK
}

\section{Reimagining Primary Health Care after COVID-19}

\author{
Athapattu P, Editor, College of Medical Administrators of Sri Lanka
}

\begin{abstract}
With the COVID-19 pandemic, policy makers, health professionals and ordinary citizens in most countries understood that business as usual in health care is no longer an option. Health systems need transformation on the scale of the crisis itself. COVID-19 has created a once-in-a-generation opportunity for sweeping systemic change backed by bold public investment and supported by broad social demand. The health care model that can drive this change is fit-for-purpose primary health care. This model is anchored in the values and lessons of the historical PHC movement. And it is reimagined for a world in which the pandemic has challenged much of what we thought we knew.
\end{abstract}

Nearly half a century after the Alma Ata Conference, hasn't the world talked enough about PHC? Clearly not, because PHC's proven benefits have still not reached hundreds of millions of people who urgently need them. And because, when COVID struck, PHC's power to protect communities in health emergencies was not used. We need to keep talking about PHC. But above all we need to walk the talk fast. The distinctive strengths of PHC are vital to "build back better" in health after the pandemic. Countries that choose the path of ambitious PHC reform will reap powerful rewards: through lower health care costs, more resilient systems, stronger human capital, increased health literacy, higher economic productivity, and above all, longer, healthier and more satisfying lives for people.

There are well functioning primary care services in the preventive care system in Sri Lanka. However, it is not seen in the curative sector to the same extent. Therefore, the Government of Sri Lanka has initiated to implement operational frame work introduced by the World Health organization on 'Primary health care: transforming vision to action' which includes fourteen levers identified as necessary for translating the commitments made in the Astana Declaration into actions and would in turn support towards the progress of strengthening primary health care. Among the core strategic levers, political commitment and leadership, governance and policy frameworks play a major role for strengthening primary health care. The 'Policy on healthcare delivery for universal health coverage' in Sri Lanka was approved by the Cabinet of Ministers in 2018, and stands as the foundation for strengthening primary care in Sri Lanka. The 'Essential Services Package' has been launched in 2019, introducing the services to be provided at each level of care. Funding and allocation of resources is another core strategic lever, where Sri Lanka is funded by the World Bank and Asian Development Bank through "Primary health care System Strengthening Project" and the "Health System Enhancement Project" respectively to improve primary care in the country, complementing the allocations of the Government of Sri Lanka. The fourth core strategic lever, 'engagement of communities and other stakeholders', is of utmost importance, and empowers the communities to work towards their own health. 'Friends of services committees' have been established in primary care hospitals in Sri Lanka with the representation of community and religious leaders in the community and grievance redress mechanisms have also been introduced.

Improving trust on the services provided is one of the major contribution to increase utilization of frontline primary health care services. Improving the quality of services provided by Primary Medical Care Institutions in turn would improve the continuity of services with improved follow up and referral systems. This would reduce the overcrowding at secondary and tertiary level health care institutions, enabling them to focus better on specialized care. The lesson learnt from COVID 19 pandemic is an eye-opener for healthcare managers to pay attention on patient centered comprehensive primary care at the community level.

Even though, Sri Lanka as a developing country, has been taking many important initiatives to re-orient its primary care system, the driving force seems to be inadequate yet. Therefore, as Medical Managers in the Sri Lanka's health system, it is our joint responsibility to facilitate implementation of reforms in the primary health care system in order to deliver a high quality health care with improved responsiveness. Let us join our hands to walk the talk to put our Vision in to action.

In this backdrop, the College of Medical Administrators of Sri Lanka is publishing the Volume 22 September 2021, of the Journal of Medical Administration consisting of research articles mainly focusing on describing the effects of the COVID 19 pandemic on Sri Lanka's health system in various angles which will be undoubtedly useful for healthcare managers to further strengthen the health care services in an evidence based manner. 